\title{
Optimal Transmission of a Set of Discrete-Time Gaussian Signals through Channels with Feedlback
}

\author{
Yoshiki Takeuchi \\ Dept. of Information Science, Osaka Univ. of Education \\ 4-698-1 Asahigaoka, Kashiwara City, Osaka 582-8582, Japan \\ Tel. +81-729-78-3669, Fax. +81-729-78-3554 \\ e-mail:takeuti@cc.osaka-kyoiku.ac.jp
}

\begin{abstract}
We consider the problem of optimal transmission of correlated Gaussian signals through a set of parallel channels with feedback. We send the signals using a linear encoding with output feedback. It is well-known that the optimal output feedback which minimizes the power of the encoded signal is given by the least-squares estimate of the linear term. Under a constraint on the total transmission power, we consider the problem of computing a set of gains for the channels which maximizes the mutual information between the output and the signal.
\end{abstract}

\section{INTRODUCTION}

For Gaussian parallel channels with feedback, there are a number of studies on transmission problems. ${ }^{[1]-[9]}$ Ihara $^{[1]}$ considered the case where the signal is a scalar random variable and the coding is liner in the signal. $\mathrm{He}$ showed that the optimal feedback signal which is used to generate the encoded signal is the least-squares estimate of the linear term and that the optimal encoded signal is the estimation error multiplied by a gain coefficient. This result is generalized by Liptser and Shiryayev ${ }^{[2]}$ to the case where the signal is a scalar Gaussian stochastic process which is given by a solution of a linear stochastic differential equation.

For the corresponding multi-dimensional case with parallel transmission channels, it was shown by Kunita ${ }^{[3]}$ that the optimal encoded signal is still the least-squares estimation error multiplied by a gain matrix. We have been, therefore, concerned with the problem of computing the optimal gain matrix which minimizes the least-squares estimation error. ${ }^{[4],[6],[7]}$ All the above works are concerned with continuous-time models. For continuous-time models, we have already considered the non-stationary case $^{[8]}$ and the stationary case ${ }^{[1]}$. For the stationary case, we obtained an analytical solution. Especially, for the case where the number of transmission channels is equal to that of the correlated Gaussian signals the optimal gain is given by a very simple form. ${ }^{[7]}$

In this paper, we consider the stationary case of the problem for discrete-time transmission models by a linear encoding of the signal. Similarly as in the continuoustime case, the optimal feedback in the linear encoding is given by such a condition that we have the innovations process as the output of the channels. When the optimal feedback is used in the discrete-time problem, however, the transmission power does not have a direct relation to the mutual information whereas in the continuous-time problem, there is a relation that the latter is always half of the former. ${ }^{[1], ~[2] ~ T h e r e f o r e, ~ u n d e r ~ a ~ c o n s t r a i n t ~ o n ~ t h e ~}$ transmission power, we consider the problem of maximizing the mutual information with respect to the channel gains. We will show that the optimal set of the gains is such that the covariance of the gained signal is diagonal and is proportional to an identity matrix. A set of equations is obtained by which we can compute the optimal set of the gains as well as the covariance matrices of the predicted and filtered estimates of the signal.

Mathematical symbols, in this paper, are used in the following way. The Euclidean norm is $|\cdot|$. The triplet $(\Omega, \mathscr{F}, P)$ is a complete probability space where $\Omega$ is a sample space with elementary events $\omega, \mathscr{F}$ is a $\sigma$-algebra of subsets of $\Omega$, and $P$ is a probability measure. $\mathrm{E}\{\bullet\}$ denotes the expectation and $E\{\bullet \mid \mathscr{G}, \mathscr{G} \subset \mathscr{F}$ the conditional expectation, given $\mathscr{G}$, with respect to $P . \sigma\{\cdot\}$ is the minimal sub- $\sigma$-algebra of $\mathscr{F}$ with respect to which the family of $\mathscr{F}$-measurable sets or random variables $\{\bullet\}$ is measurable. It is assumed that all random variables and stochastic processes are $\mathscr{F}$-measurable.

\section{PROBLEM FormuLATION AND PRELIMINARY}

Let us consider a set of transmission channels with feedback as shown in Fig. 1. We assume that signal $x \equiv\left\{x_{t}(\omega) ; t=0,1, \cdots\right\}$ is an $n$-dimensional Gaussian stochastic process given by

$$
x_{t+1}(\omega)=A(t) x_{t}(\omega)+G(t) w_{t}(\omega), x_{0}(\omega)=x^{0}(\omega),
$$

where $x^{0}(\omega)$ is an $n$-dimensional Gaussian random vector with mean $\hat{x}^{0}$ and covariance $Q^{0}$, and $w \equiv\left\{w_{t}(\omega)\right.$; 
$t=0,1, \cdots\}$ is a $d$-dimensional standard Gaussian white noise sequence which is independent of $x^{0}(\omega)$.

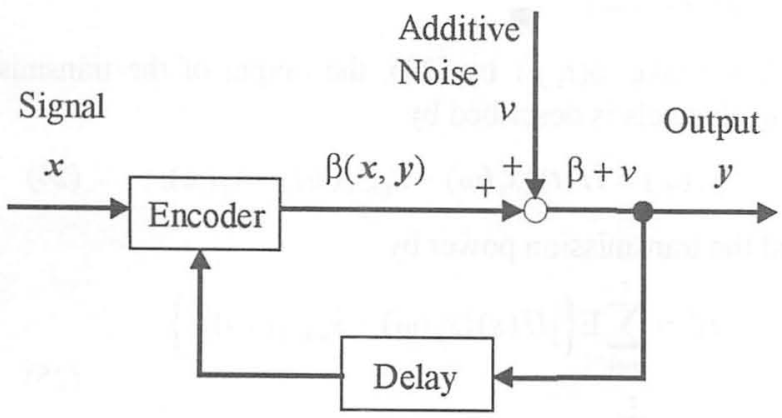

Fig. 1 Transmission channels with feedback.

When we send $x$ through the set of $m$-parallel channels, we employ an encoding scheme. Namely, we send an encoded signal $\beta \equiv\left\{\beta_{t}(x, y) ; t=1,2, \cdots\right\}$ which is a causal function of both signal $\boldsymbol{x}$ and output $y \equiv\left\{y_{t}(\omega)\right.$; $t=1,2, \cdots\}$.

Thus, the output of the transmission channels in Fig. 1 is described by

$$
y_{t}(\omega)=\beta_{t}(x, y)+v_{t}(\omega),
$$

where $v \equiv\left\{v_{t}(\omega) ; t=1,2, \cdots\right\}$ is an $m$-dimensional standard Gaussian white noise sequence independent of $w$ and $x^{0}(\omega)$.

An optimal transmission problem, or an optimal coding problem, is the one to determine $\beta$ optimally. Let $I_{t}(x, y)$ denote the mutual information between $x^{t} \equiv$ $\left\{x_{s}(\omega) ; s=0,1, \cdots, t\right\}$ and $y^{t} \equiv\left\{y_{s}(\omega) ; s=1,2, \cdots, t\right\}$, and $P_{0}^{t}$ the transmission power up to $t$, i.e.,

$$
P_{0}^{t} \triangleq \sum_{s=1}^{t} \mathrm{E}\left\{\left|\beta_{s}(x, y)\right|^{2}\right\} .
$$

It is desirable to select $\beta$ in such a way that we have output $y$ for which $I_{t}(x, y)$ takes a value as large as possible. On the other hand, it is usually the case that $P_{0}^{t}$ cannot exceed a value, say $P^{*}(t)$, which is determined by the restriction on the transmission channels. Then, the optimal transmission problem is described by

[Optimal Transmission Problem] Find $\beta$ such that

$$
I_{t}(x, y) \rightarrow \max .,
$$

subject to the power condition:

$$
P_{0}^{t} \leq P^{*}(t)
$$

where $P^{*}(t)$ is the maximum admissible transmission power up to time $t$.

As it is well-known, the solution of the above problem is also that of
[The Dual Problem] Find $\beta$ such that

$$
P_{0}^{t} \rightarrow \min , \text {, }
$$

subject to the condition on the capacity:

where

$$
I_{t}(x, y) \leq C^{*}(t),
$$

$$
C^{*}(t) \triangleq \max _{\substack{\beta \\ P_{0}^{t} \leq P^{*}(t)}} I_{t}(x, y) .
$$

In this paper, we are concerned with a linear coding problem where $\beta$ is given by

$$
\beta_{t}(x, y)=H(t) x_{t}(\omega)-\phi(t, y) .
$$

Since $\phi(t, y)$ must be a non-anticipative function of $y$, we have

$$
\phi(t, y)=\phi\left(t, y_{1}, \cdots, y_{t-1}\right)
$$

namely, $\phi(t, y)$ should be a $\mathscr{Y}_{t-1}$-measurable random vector, where

$$
\mathscr{Y}_{t} \triangleq \sigma\left\{y_{s}(\omega) ; s=1,2, \cdots, t\right\} .
$$

As it is also well-known, $\phi(t, y)$ does not affect the information obtained from $y$.

«Theorem 1 »» Let $y^{*} \equiv\left\{y_{t}{ }^{*}(\omega) ; t=1,2, \cdots\right\}$ denote the value of $y$ when $\phi(t, y) \equiv 0$, i.e.,

$$
y_{t}^{*}(\omega)=H(t) x_{t}(\omega)+v_{t}(\omega) .
$$

Then, we have (i) and (ii) below.

(i) There is the informational equivalence between $y$ and $y^{*}$, i.e., we have

$$
\mathscr{Y}_{t}=\mathscr{Y}_{t}^{*}, \quad t=1,2, \cdots,
$$

where

$$
\mathscr{Y}_{t}^{*} \triangleq \sigma\left\{y_{s}^{*}(\omega) ; s=1,2, \cdots, t\right\} .
$$

(ii) $I_{t}(x, y)$, i.e., the mutual information between $x^{t}$ and $y^{t}$, does not depend on $\phi(s, y), s \leq t$ and is given by

$$
I_{t}(x, y)=\frac{1}{2} \sum_{s=1}^{t} \log \left\{\operatorname{det}\left|H(s) Q_{s \mid s-1} H^{\prime}(s)+I\right|\right\} .
$$

where

$$
Q_{s \mid s-1} \triangleq E\left\{\left[x_{s}(\dot{\omega})-\hat{x}_{s \mid s-1}(\omega)\right]\left[x_{s}(\omega)-\hat{x}_{s \mid s-1}(\omega)\right]^{\prime}\right\},
$$
and

$$
\hat{x}_{s \mid s-1}(\omega) \triangleq E\left\{x_{s}(\omega) \mid \mathscr{Y}_{s-1}\right\} \text {. }
$$

(Proof ) The proof of (i) is trivial and can be found in [11]. Also, (ii) is proved rather trivially. Note that

$$
I_{t}(x, y)=E\left\{\log \frac{p\left(x^{t}, y^{t}\right)}{p\left(x^{t}\right) p\left(y^{t}\right)}\right\},
$$

where $p\left(x^{t}\right), \quad p\left(y^{t}\right)$ and $p\left(x^{t}, y^{t}\right)$ are the probability density and the joint probability density functions of $x^{t}$, 
$y^{t}$ and $\left(x^{t}, y^{t}\right)$. It can be easily seen by the Bayes' formula that

$$
p\left(x^{t}, y^{t}\right)=\prod_{s=1}^{t} p\left(y_{s} \mid y^{s-1}, x^{t}\right) p\left(x^{t}\right),
$$

and $p\left(y_{s} \mid y^{s-1}, x^{t}\right) \sim \mathscr{N}^{m}\left(\beta_{s}(x, y), I\right)$, where $\mathscr{N}^{m}(\xi, \Sigma)$ denotes the $m$-dimensional Gaussian distribution with mean vector $\xi$ and covariance matrix $\Sigma$. Similarly, we also have

$$
p\left(y^{t}\right)=\prod_{s=1}^{t} p\left(y_{s} \mid y^{s-1}\right),
$$

and $p\left(y_{s} \mid y^{s-1}\right) \sim \mathcal{N}^{m}\left(\hat{\beta}_{s \mid s-1}, H(s) Q_{s \mid s-1} H^{\prime}(s)+I\right)$, where

$$
\hat{\beta}_{s \mid s-1} \triangleq E\left\{\beta_{s}(x, y) \mid \mathscr{Y}_{s-1}\right\}=H(s) \hat{x}_{s \mid s-1}-\phi(s, y) .
$$

Hence, substitution of (19) and (20) into (18) yields (15) immediately. This completes the proof.

Now, the optimal transmission problem is that of finding $\varphi^{t} \equiv\{\phi(s, y) ; s=0,1, \cdots, t\}$ and $\boldsymbol{H}^{t} \equiv\{H(s) ; s=0,1, \cdots$, $t\}$ for which we have (4) and (5). By Theorem 1, the dual problem given by (6) and (7) is divided into the following two sub-problems:

[Problem S1] For a fixed $\boldsymbol{H}^{t}$, find $\varphi^{t}$ such that

$$
P_{0}^{t} \rightarrow \min \text {. }
$$

[Problem S2] For the solution $\varphi^{t}=\varphi_{*}^{t}$ of Problem S1, find $H^{t}$ such that

$$
P_{0}^{t} \rightarrow \min .
$$

subject to the condition on the capacity:

$$
I_{t}(x, y) \leq C^{*}(t) .
$$

As for Problem S1, it can be seen from (3) and (9) that $\varphi^{t} \equiv\{\phi(s, y) ; s=0,1, \cdots, t\}$ is optimal when

$$
P_{0}^{t}=\sum_{s=1}^{t} \mathrm{E}\left\{\left|H(s) x_{s}(\omega)-\phi(s, y)\right|^{2}\right\},
$$

is minimized. According to the least-squares estimation theory, the optimal selection of $\phi(s, y), s \leq t$ is given by the following theorem.

«Theorem 2» For all $t$, the solution of Problem S1 is given by

$$
\phi(s, y)=H(s) \hat{x}_{s \mid s-1}(\omega), \quad s=1,2, \cdots, t,
$$

where $\hat{x}_{s \mid s-1}(\omega)$ is the prediction of $x_{s}(\omega)$ defined by (17).

[Remark 1] It should be noted that the optimal $\varphi^{t}$ is such that $y^{t}$ becomes the innovations process of the "observation" $y^{*}$ given by (12). ${ }^{[2],[5]}$
[Remark 2] It should be noted that the optimization in Problem S1 is not of multi-stage but of stage-wise. In fact, the value of $P_{s}^{t} \triangleq P_{0}^{t}-P_{0}^{s-1}, s \leq t$ is independent of $\phi(\tau, y), \tau \leq s-1$.

If we take $\phi(t, y)$ by (23), the output of the transmission channels is described by

$$
y_{t}(\omega)=H(t)\left\{x_{t}(\omega)-\hat{x}_{t \mid t-1}(\omega)\right\}+v_{t}(\omega),
$$

and the transmission power by

$$
\begin{aligned}
P_{0}^{t} & =\sum_{s=1}^{t} \mathrm{E}\left\{\left|H(s)\left\{x_{s}(\omega)-\hat{x}_{s \mid s-1}(\omega)\right\}\right|^{2}\right\} \\
& =\sum_{s=1}^{t} \operatorname{tr}\left[H(s) Q_{s \mid s-1} H^{\prime}(s)\right] .
\end{aligned}
$$

Now, by taking the dual of Problem S2 again, the rest half of the problem is described by

[Problem S2' (Optimal Selection of $\left.\left.H^{t}\right)\right]$ Find $H^{t} \equiv$ $\{H(s) ; s=0,1, \cdots, t\}$ such that

$$
I_{t}(x, y)=\frac{1}{2} \sum_{s=1}^{t} \log \left\{\operatorname{det}\left|H(s) Q_{s \mid s-1} H^{\prime}(s)+I\right|\right\} \rightarrow \max .
$$

subject to the power condition:

$$
P_{0}^{t}=\sum_{s=1}^{t} \operatorname{tr}\left[H(s) Q_{s \mid s-1} H^{\prime}(s)\right] \leq P^{*}(t) .
$$

Let $\hat{x}_{t \mid t}(\omega)$ and $Q_{t \mid t}$ denote respectively the leastsquares estimation of $x_{t}(\omega)$ given $\mathscr{y}_{t}$ and its error covariance matrix, i.e.,

$$
\hat{x}_{t \mid t}(\omega) \triangleq E\left\{x_{t}(\omega) \mid \mathscr{Y}_{t}\right\},
$$

and

$$
Q_{t \mid t} \triangleq E\left\{\left[x_{t}(\omega)-\hat{x}_{t \mid t}(\omega)\right]\left[x_{t}(\omega)-\hat{x}_{t \mid t}(\omega)\right]^{\prime}\right\} .
$$

In what follows, we are concerned with the stationary case where $A(t) \equiv A, G(t) \equiv G, H(t) \equiv H$, and the error covariance matrices have the properties: $Q_{t \mid t} \rightarrow Q$ and $Q_{t \mid t-1} \rightarrow Q^{-}$as $t \rightarrow \infty$. As it is well-known, the steady-state covariance matrices $Q$ and $Q^{-}$are determined by

$$
Q=Q^{-}-Q^{-} H^{\prime}\left(H Q^{-} H^{\prime}+I\right)^{-1} H Q^{-},
$$

and

$$
Q^{-}=A Q A^{\prime}+G G^{\prime} .
$$

We will assume that $A, G$ and $H$ are such matrices for which (29) and (30) have a positive definite solution. For this case, the above problem reduces as follows.

[Problem 1 (Optimal Selection of $H)$ ] Find $H \in R^{m \times n}$ such that

$$
\bar{I}(x, y)=\frac{1}{2} \log \left\{\operatorname{det}\left|H Q^{-} H^{\prime}+I\right|\right\} \rightarrow \max .
$$


subject to

$$
\bar{P}_{0}=\operatorname{tr}\left[H Q^{-} H^{\prime}\right] \leq p,
$$

(29) and (30).

In (31) and (32), $\bar{P}_{0}$ and $\bar{I}(x, y)$ are the transmission power and the mutual information per unit time, respectively, and $p$ is the maximum admissible value of the former.

\section{OPTIMAL SELECTION OF $H$}

A basic solution of Problem 1 is given by the following two theorems.

«Theorem 3» Assume that $G G^{\prime}>0$. Then, $H \in R^{m \times n}$ is optimal when all eigen-values of $H Q^{-} H^{\prime}$ are equal to $\mathrm{p} / \mathrm{m}$ and $H Q^{-} H^{\prime}$ is given by

$$
H Q^{-} H^{\prime}=\frac{p}{m} I \text {. }
$$

Furthermore, for the optimal value of $H$, we have

$$
H Q H^{\prime}=\frac{p}{p+m} I .
$$

«Theorem 4» Assume that $G G^{\prime}>0$. The solution of Problem 1 is given by the following set of equations.

$$
\begin{gathered}
Q^{-}=A Q A^{\prime}+G G^{\prime} \\
Q=\left(Q^{-}\right)^{1 / 2} K\left(Q^{-}\right)^{1 / 2}, \\
K \triangleq\left(I+\frac{p}{m} U\right)^{-1}, \\
H^{\prime} H=\frac{p}{m}\left(Q^{-}\right)^{-1 / 2} U\left(Q^{-}\right)^{-1 / 2},
\end{gathered}
$$

where $U \in R^{n \times n}$ is any symmetric matrix whose $m$ eigenvalues are 1 and the rest are 0 , i.e.,

$$
U=\tilde{U} \tilde{U}^{\prime}, \quad \tilde{U} \in \boldsymbol{R}^{n \times m}, \quad \tilde{U}^{\prime} \tilde{U}=I .
$$

Let $S \triangleq\left(Q^{-}\right)^{1 / 2}$. Then, the result of Theorem 4 is described in a more convenient form as follows.

«Theorem 5» Assume that $G G^{\prime}>0$. Let $S$ denote a solution of the quadratic matrix equation:

$$
S^{2}=A S K S A^{\prime}+G G^{\prime},
$$

where

$$
K \triangleq\left(I+\frac{p}{m} U\right)^{-1}
$$

Then, the solution of Problem 1 is given by the following set of equations:

$$
\begin{gathered}
Q^{-}=S^{2}, \\
Q=S K S, \\
H^{\prime} H=\frac{p}{m} S^{-1} U S^{-1},
\end{gathered}
$$

where $U \in R^{n \times n}$ is given by (38).
[Remark 3] As we see from the above theorems, especially from (43) and (38), the optimal $H \in R^{m \times n}$ is not unique but has a large degree of freedom if $m<n$. It should be noted, however, that this is consistent with the result for the continuous-time case ${ }^{[7]}$ where $H$ is any matrix which satisfies the power condition at the upper bound.

[Remark 4] It should be noted that if $A \in R^{n \times n}$ is a stable matrix, then we always have a positive definite solution of (39).

\section{Proofs OF THEOREMS}

In this section, we will give proofs of the theorems presented in the previous section. First, the proof of Theorem 3 is straight-forward by using the following lemma.

[Lemma 1] For all $H \in R^{m \times n}$, there exists $\tilde{H} \in R^{m \times n}$ such that $\tilde{H} Q^{-} \tilde{H}^{\prime}$ is a diagonal matrix, i.e.,

$$
\tilde{H} Q^{-} \tilde{H}^{\prime}=\Xi, \quad \Xi=\operatorname{diag}\left(\xi_{1}, \xi_{2}, \cdots, \xi_{m}\right),
$$

and that we have the same values of $\bar{I}(x, y), P_{0}, Q^{-}$and $Q$ for both $H$ and $\tilde{H}$.

(Proof) For any $H \in R^{m \times n}$ with $\operatorname{rank}[H]=m$, let us set as

$$
H Q^{-} H^{\prime}=\Gamma \Xi \Gamma^{\prime}, \quad \Xi=\operatorname{diag}\left(\xi_{1}, \xi_{2}, \cdots, \xi_{m}\right),
$$

where $\Gamma$ is an orthogonal matrix in $R^{m \times m}$, i.e., $\Gamma^{\prime} \Gamma=$ $\Gamma \Gamma^{\prime}=I$. Also, let $\tilde{H} \triangleq \Gamma^{\prime} H$. Then, we can easily see the above statement by (31), (32), (29) and (30).

(Proof of Theorem 3) By Lemma 1, we can assume, without loss of generality, that $H Q^{-} H^{\prime}$ is a diagonal matrix, i.e.,

$$
H Q^{-} H^{\prime}=\Xi, \quad \Xi=\operatorname{diag}\left(\xi_{1}, \xi_{2}, \cdots, \xi_{m}\right),
$$

Hence, we can replace (31) and (32) by

$$
\operatorname{det}\left|H Q^{-} H^{\prime}+I\right|=\prod_{i=1}^{m}\left(\xi_{i}+1\right) \rightarrow \max .,
$$

and

$$
p+m \geq \operatorname{tr}\left[H Q^{-} H^{\prime}\right]+\operatorname{tr}[I]=\sum_{k=1}^{m}\left(\xi_{i}+1\right)
$$

respectively. Thus, we have

$$
\xi_{i}+1=\frac{p+m}{m}, \quad i=1,2, \cdots, m,
$$

which is equivalent to

$$
\xi_{i}=\frac{p}{m}, \quad i=1,2, \cdots, m .
$$

Combination of (46) and (50) implies (33). Then, substitution of (33) into (29) multiplied by $H$ from left and $H^{\prime}$ from right yields (34). This completes the proof.

Now, let us proceed to the proof of Theorem 4. The 
proof is based on the following lemma.

[Lemma 2] Let $X \in R^{n \times n}$ be defined by

$$
X \triangleq\left(Q^{-}\right)^{1 / 2} H^{\prime} H\left(Q^{-}\right)^{1 / 2},
$$

Then, we have

(i) $X$ is a solution of $X^{2}=\frac{p}{m} X$.

(ii) The solution is given by $X=\frac{p}{m} U$, where $U \in R^{n \times n}$ is given by (38).

(Proof ) First, (i) is easily seen from (33) as

$$
X^{2}=\left(Q^{-}\right)^{1 / 2} H^{\prime} \cdot H Q^{-} H^{\prime} \cdot H\left(Q^{-}\right)^{1 / 2}=\frac{p}{m} X .
$$

To show (ii), note that we can write $X=\tilde{U} \Theta \tilde{U}^{\prime}$, where $\Theta=\operatorname{diag}\left(\theta_{1}, \theta_{2}, \cdots \theta_{m}\right) \in R^{m \times m}, \quad \theta_{i}>0, \quad i=1,2, \cdots, m$, since $\operatorname{rank}[H]=\operatorname{rank}[X]=m$. Then, from (i), we have

$$
\theta_{i}^{2}=\frac{p}{m} \theta_{i}, \quad \theta_{i}>0, \quad i=1,2, \cdots, m .
$$

Hence, we have $\theta_{i}=\frac{p}{m}, i=1,2, \cdots, m$, and which implies (ii). This completes the proof.

(Proof of Theorem 4) By Lemma 2, we now have

$$
X \triangleq\left(Q^{-}\right)^{1 / 2} H^{\prime} H\left(Q^{-}\right)^{1 / 2}=\frac{p}{m} U .
$$

Hence, we have (37). Using the well-known formula of matrix inversion to (29), we have

$$
\left(Q^{-}\right)^{-1}+H^{\prime} H=Q^{-1} \text {. }
$$

Then, substitution of (37) into (55) yields

$$
\left(Q^{-}\right)^{-1}+\frac{p}{m}\left(Q^{-}\right)^{-1 / 2} U\left(Q^{-}\right)^{-1 / 2}=Q^{-1},
$$

and which implies

$$
\left(Q^{-}\right)^{-1 / 2}\left(I+\frac{p}{m} U\right)\left(Q^{-}\right)^{-1 / 2}=Q^{-1} .
$$

By taking the inverse of (57), we obtain (35) and (36). This completes the proof.

\section{CONSIDERATIONS FOR NUMERICAL COMPUTATIONS}

By Theorem 5, if we have solution $S$ of (39), we can compute optimal $H$ from (43). The solution of (39) is easily computed by the following recursive approximation scheme.

\section{[A Recursive Algorithm to compute $S$ ]}

(i) Set the initial value by $S_{0}=I$.

(ii) Update $S_{i}$ and $Q_{i}^{-}$by

$$
Q_{i}^{-}=A S_{i-1} K S_{i-1} A^{\prime}+G G^{\prime}, \quad i=1,2, \cdots
$$

and

$$
S_{i}=\left(Q_{i}^{-}\right)^{1 / 2} .
$$

(iii) If $S_{i}$ and $Q_{i}^{-}$have reached an equilibrium state, i.e., if we have $S_{i} \cong S_{i-1}$ and $Q_{i}^{-} \cong Q_{i-1}^{-}$, set as $S=S_{i}$ and $Q^{-}=Q_{i}^{-}$.

In the numerical calculations in the next section, the above algorithm will be used to obtain the solution of (39).

\section{A NUMERICAL EXAMPLE}

In this section, we will give an illustrative example for a three dimensional system and three cases of value of $m$.

[Example 1] Let us consider 3-dimensional system $(n=3)$ :

and

$$
A=\left[\begin{array}{ccc}
0.5 & 0.3 & 0.1 \\
0.2 & 0.4 & 0.2 \\
0.1 & 0.5 & 0.6
\end{array}\right], \quad G=\left[\begin{array}{ccc}
0.5 & 0 & 0 \\
0 & 0.5 & 0 \\
0 & 0 & 0.5
\end{array}\right],
$$

$$
p=2 .
$$

(Case 1: $m=n=3$ ) For this case, by (38), we have $U=I$. Namely, $U$ is unique and is an identity matrix. By (40)-(43) together with the recursive algorithm (58) and (59), we obtained the following values which were checked to meet the requirement for the optimal solution.

$$
\begin{gathered}
Q^{-}=\left[\begin{array}{lll}
0.346 & 0.076 & 0.103 \\
0.076 & 0.323 & 0.114 \\
0.103 & 0.114 & 0.451
\end{array}\right], Q=\left[\begin{array}{lll}
0.208 & 0.045 & 0.062 \\
0.045 & 0.194 & 0.068 \\
0.062 & 0.068 & 0.271
\end{array}\right], \\
H^{\prime} H=\left[\begin{array}{ccc}
2.123 & -0.356 & -0.397 \\
-0.356 & 2.322 & -0.504 \\
-0.397 & -0.504 & 1.697
\end{array}\right],
\end{gathered}
$$

and

$$
H=\sqrt{\frac{p}{m}} \tilde{U}^{\prime}\left(Q^{-}\right)^{-1 / 2}=\left[\begin{array}{ccc}
0.582 & -1.430 & 0.514 \\
1.238 & 0.185 & -0.888 \\
0.502 & 0.493 & 0.803
\end{array}\right] .
$$

(Case 2: $m=2<n$ ) For this case, the value of $U$ has a large degree of freedom. We made the computations for the following set of values of $U$ :

(i) $U=\operatorname{diag}(1,1,0)$,

(ii) $U=\operatorname{diag}(1,0,1)$

(iii) $U=\operatorname{diag}(0,1,1)$.

For example, for case (iii), we obtained

$$
\begin{gathered}
Q^{-}=\left[\begin{array}{lll}
0.400 & 0.099 & 0.119 \\
0.099 & 0.329 & 0.110 \\
0.119 & 0.110 & 0.427
\end{array}\right], Q=\left[\begin{array}{ccc}
0.393 & 0.074 & 0.087 \\
0.074 & 0.168 & 0.058 \\
0.087 & 0.058 & 0.217
\end{array}\right], \\
H^{\prime} H=\left[\begin{array}{ccc}
0.081 & -0.320 & -0.287 \\
-0.320 & 3.430 & -0.752 \\
-0.287 & -0.752 & 2.658
\end{array}\right],
\end{gathered}
$$

and 


$$
H=\sqrt{\frac{p}{m}} \tilde{U}^{\prime}\left(Q^{-}\right)^{-1 / 2}=\left[\begin{array}{ccc}
-0.066 & 1.691 & -1.014 \\
-0.277 & 0.755 & 1.277
\end{array}\right] .
$$

(Case 3: $m=1<n$ ) For this case, the value of $U$ also has a large degree of freedom. We made the computations for the following set of values of $U$ :

(i) $U=\operatorname{diag}(1,0,0)$,

(ii) $U=\operatorname{diag}(0,1,0)$,

(iii) $U=\operatorname{diag}(0,0,1)$.

For example, for case (iii), we obtained

$$
\begin{gathered}
Q^{-}=\left[\begin{array}{lll}
0.468 & 0.165 & 0.209 \\
0.165 & 0.394 & 0.196 \\
0.209 & 0.196 & 0.535
\end{array}\right], Q=\left[\begin{array}{lll}
0.454 & 0.152 & 0.143 \\
0.152 & 0.382 & 0.131 \\
0.143 & 0.131 & 0.204
\end{array}\right], \\
H^{\prime} H=\left[\begin{array}{ccc}
0.155 & 0.165 & -0.854 \\
0.165 & 0.177 & -0.912 \\
-0.854 & -0.912 & 4.710
\end{array}\right],
\end{gathered}
$$

and

$$
H=\sqrt{\frac{p}{m}} \tilde{U}^{\prime}\left(Q^{-}\right)^{-1 / 2}=\left[\begin{array}{lll}
0.394 & 0.420 & -2.170
\end{array}\right] .
$$

The values of $\bar{I}(x, y)$ and $\operatorname{tr}[Q]$ for the above three cases are summarized in Table 1. From Table 1, we notice that the value of $\operatorname{tr}[Q]$, namely, the lest-squares estimation error, does depend on the selection of $U$ even if they produce the same value of the mutual information: $\bar{I}(x, y)$. Thus, from the view point of the performance of the least-squares estimation of the signal, there remains

\begin{tabular}{|c|c|c|c|c|}
\hline$p$ & $m$ & $U$ & $\bar{I}(x, y)$ & $\operatorname{tr}[Q]$ \\
\hline \multirow{7}{*}{2} & 3 & $I=\operatorname{diag}(1,1,1)$ & 0.7662 & 0.6720 \\
\hline & \multirow{3}{*}{2} & $\operatorname{diag}(1,1,0)$ & \multirow{3}{*}{0.6931} & 0.9830 \\
\hline & & $\operatorname{diag}(1,0,1)$ & & 0.8130 \\
\hline & & $\operatorname{diag}(0,1,1)$ & & 0.7779 \\
\hline & \multirow{3}{*}{1} & $\operatorname{diag}(1,0,0)$ & \multirow{3}{*}{0.5493} & 1.6270 \\
\hline & & $\operatorname{diag}(0,1,0)$ & & 1.1933 \\
\hline & & $\operatorname{diag}(0,0,1)$ & & 1.0400 \\
\hline
\end{tabular}
a problem of optimal selection of $U$ except for the case $m=n$ where $U$ is determined uniquely.

Table 1. The values of $\bar{I}(\boldsymbol{x}, \boldsymbol{y})$ and $\operatorname{tr}[Q]$.

\section{CONCLUding REMARKS}

In this paper, we have considered the optimal transmission of multi-dimensional Gaussian signals through parallel channels with feedback. We considered the stationary case, and obtained a set of equations to compute the optimal coefficient matrix for the linear encoding of the signal. It was shown that the value of the optimal coefficient matrix is not unique when the number of the transmission channel is less than that of the signal components.

In this paper, we are only concerned with the case where the channel noises are mutually independent and have unit variances. The result for a more general case where the channel noises are correlated and have any covariance will be reported in near future. Also, applications to the optimizations in the Kalman filter will be reported in future.

\section{REFERENCES}

[1] S. Ihara, "Coding theory in white Gaussian channel with feedback," J. Multi-variate Analysis, 4, 74/87, 1974.

[2] R. S. Liptser and A. N. Shiryayev, Statistics of Random Processes II: Applications, Springer-Verlag, New York, 1978.

[3] H. Kunita, Estimation of Stochastic Processes, Sakgyotosho, Tokyo, 1976. (In Japanese)

[4] Y. Takeuchi and T. Ishikawa, "The optimal transmission of a set of Gaussian signals through parallel channels with feedback," Proc. of 25th ISCIE SSS, Nov. 10-12, 255/260, 1993.

[5] Y. Takeuchi, "The optimal transmission of non-Gaussian signals through a noisy channel with feedback," IEEE Trans. Inform. Theory, IT-40, 5, 1624/1629, 1994.

[6] Y. Takeuchi, "The optimal transmission of correlated Gaussian n-signals through parallel m-channels with feedback," Proc. of 27th ISCIE SSS, Oct. 31 - Nov. 2, 205/210, 1995.

[7] Y. Takeuchi, "The optimal transmission of correlated Gaussian signals through parallel channels with feedback," Proc. of IEEE 35th CDC, Kobe, Japan, 2, 1890/1895, 1996.

[8] Y. Takeuchi, "An information theoretic approach to sensor allocation of Kalman-Bucy filter," Proc. of 32nd ISCIE SSS, Nov. 1 - 2, 2001.

[9] Y. Takeuchi, M. Sowa and K. Horikawa, "An information theoretic scheme for sensor allocation of linear least-squares estimation," Proc. of SICE 2002 Osaka, 248/253, 2002.

[10] Y. Takeuchi and K. Dei, "The optimal transmission of a set of Gaussian signals through parallel channels with feedback," Proc. of 34th ISCIE SSS, 78/83, 2003.

[11] Y. Takeuchi and A. Ida, "Optimal Transmission of a set of Gaussian Signals through Discrete-Time Channels with Feedback," Proc. of 35th ISCIE SSS, Ube, Oct. 3031, 2003, 118/123, 2004.

[12] Y. Takeuchi, "An information theoretic approach to optimization of linear observations for the Kalman-Bucy fiter," International Journal of Innovative Computing, Information \& Control, 1-3, 401/416, 2005.

[13] S. Ihara, Information Theory for Continuous Systems, World Scientific, 1993. 